\title{
Aprovechamiento tecnológico del almendro de india (Terminalia catappa L) para la obtención de productos alimenticios
}

\author{
Technological benefits of Indian almond (Terminalia catappa L ) \\ for the production of foodstuffs
}

\section{Utilize a tecnologia de amendoeirasindian (Terminalia catappa L) para obtenção de alimentos}

\author{
Guillermo S. Arrázola-Paternina ${ }^{1 *}$; Armando Alvis-Bermúdez ${ }^{1 *}$; \\ Irina C. Herazo-Camacho ${ }^{2 *}$ \\ 1 Ing. PhD \\ 2 Ing. MSc \\ * Grupo de Investigación procesos y Agro industria de vegetales. Programa de Ingeniería de alimentos. Facultad de \\ ingeniería. Km 3 Vía Cereté. Montería, Córdoba, Colombia \\ Email: guillermo.arrazola@ua.es
}

Recibido: enero 19 de 2013

Aceptado: 19 febrero de 2015

\section{Resumen}

La almendra del árbol, (Terminalia catappa L.) es un fruto seco que contiene nutrientes como proteína y grasa, similares a otras variedades de almendras de mayor demanda en el mercado, por lo que podría ser empleada en el aprovechamiento industrial y como alternativa nutricional. El objetivo del presente trabajo fue aprovechar tecnológicamente el fruto del almendro, mediante la obtención de harina para la elaboración de tortas y la obtención de almendras confitadas. Para la elaboración de los diferentes productos se realizó la extracción de la semilla del almendro, la cual fue sometida a operaciones unitarias, como tostado y molienda para la obtención de harinas, con la consiguiente elaboración de tortas de acuerdo a las formulaciones tradicionales, sustituyendo el $40 \%$ de harina de trigo por harina de almendras. Para la elaboración de almendras confitadas se realizó un tostado y recubrimiento de almendras con jarabes y aditivos alimentarios. Se realizó la comparación de los productos elaborados con controles o con productos comerciales y se sometieron a análisis sensorial. Los resultados organolépticos y sensoriales mostraron que las almendras confitadas y tortas de harina de almendras presentaron buena aceptabilidad por los consumidores de estos productos, constituyéndose así, en una alternativa de uso en la industria, con un importante aporte de nutrientes, sabor y aroma agradable; sin embargo presentaron diferencias significativas con los controles, en la percepción sensorial; de esta manera es posible presentar alternativas positivas como resultados de pruebas con catadores mediante la optimización de las formulaciones empleadas y la estandarización de los procesos.

Palabras claves: Harina de almendras, almendras confitadas, almendras de la India, harina de trigo, almendras para pastelería

\begin{abstract}
The West Indian almond tree (Terminalia catappa L.) produces dry fruit (a drupe, not a nut) whose seed contains nutrients such as proteins and fats, similar to other varieties of almonds having greater demand on the market; this means that it can be used in industrial exploitation and as a nutritional alternative. This work was aimed at technologically exploiting West
\end{abstract}


Indian almond fruit by obtaining flour for making cakes and obtaining candied almonds. The seed was extracted from the almond for preparing different products; it was then used in unit operations such as roasting and grinding, to obtain the flour for making cakes according to traditional recipes, $40 \%$ of wheat flour being substituted by almond flour. Preparing candied almonds involved toasting the almonds and covering them with syrup and food additives. The aforementioned products were then compared to controls or commercial products and sensory testing/tasting was evaluated. The organoleptic and sensory results showed that the candied almonds and almond flour cakes were well accepted by people consuming these products, thereby representing an alternative for use in the food industry, providing a significant source of nutrients, taste and pleasant aroma; however, there were significant differences regarding the controls in terms of sensory perception, meaning that positive alternatives could be presented, such as the results of tests with tasters by optimising the formulations used and standardising processes.

Key words: almond flour, candied almonds, West Indian almonds, wheat flour, almond pastry.

\section{Resumo}

A amêndoa (Terminalia catappa L.) é uma porca que contém quantidades de nutrientes tais como proteína e gordura, semelhante a outras variedades de amêndoas com maior procura no mercado, pelo que pode ser utilizado na utilização industrial e como uma alternativa nutricional.

O objetivo deste estudo foi utilizar a fruta da amêndoa tecnologicamente, através da obtenção de farinha para fazer bolos e obtenção de amêndoas cristalizadas. Para a preparação de diferentes produtos a semente de amêndoa foi extraída, que foi submetido a operações unitárias como a torrefacção e moagem para obtenção de farinha, tornando assim bolos de acordo com formulações tradicionais, em substituição de $40 \%$ de farinha de trigo com farinha de amêndoa. Para preparar amêndoas torradas cristalizadas e revestimento foi feito com amêndoas xarope e aditivos alimentares. Comparando-se os produtos processados foi realizado com controles ou produtos comerciais e foi submetido à análise sensorial. Os resultados mostraram que organolépticas amêndoas revestidas de açúcar e bolos de farinha de amêndoa apresentaram boa aceitabilidade pelos consumidores desses produtos, constituindo, assim, uma alternativa para uso na indústria, um nutriente importante, sabor e aroma agradável; poréma presentaram diferenças significativas de controles na percepção sensorial; desta forma é possívela presentar alternativas positivas, tais como resultados de testes comprovadores, otimizando formulações utilizadas e a padronização dos processos.

Palavras-chave: Farinha, amêndoas, amêndoas cristalizadas, amêndoa Índia, farinha de trigo, amêndoa doces.

\section{Introducción}

La almendra (Terminalia catappa L) es un fruto drupáceo, carnoso y ovoide, que contiene una semilla o núcleo de sabor y aroma agradable al paladar, de color blanco cubierta por una cáscara muy delgada de color café rojizo (González et al., 2005; Hernández et al., 2003).

La harina obtenida de almendra puede ser utilizada en productos de alto valor nutritivo debido a su alto contenido de proteínas el cual ha sido reportado por algunos autores entre 23 al 26\% (Matos et al., 2009; Arrazola et al., 2008); su valor nutricional sumado a su exquisito sabor permite que las almendras puedan ser empleadas en diversas formas, como almendras enteras, laminadas, rodajas, confitadas, o también ser utilizado en muchos productos de panadería como galletas, tortas y pasteles (Fasoli et al., 2011).

La sustitución parcial de la harina de trigo por harina de almendras en las formulaciones tradicionales de productos de panadería, permitiría mejorar el valor nutritivo de estos productos, su reemplazo parcial podría ser una alternativa para disminuir el uso de materias primas tradicionales que son en gran parte importadas y con precios ascendentes (Henao y Aristizábal, 2009). Otra alternativa de aprovechamiento tecnológico, es la obtención de grageas o almendras recubiertas (confitadas), siendo una importante área de desarrollo en especial por la industria de confitería (Onwulata, 2005). El recubrimiento duro de grageas está destinado a conseguir una capa crujiente y azucarada, en el caso de almendras permite protegerlas contra la rancidez y además contribuir a mejorar su sabor y sus características organolépticas (Mejorado, 2006; Roquette y Cabinet, 2006; Ando et. al., 2007).

El objetivo del presente trabajo fue buscar alternativas al aprovechamiento tecnológico de almendras (Terminalia catappa) en la elaboración de tortas y la obtención de almendras confitadas.

\section{Materiales y métodos}

Recolección de almendras. Los frutos del almendro de la India, fueron recolectados en el departamento de Córdoba, donde crece de manera silvestre. Para elaborar los diversos productos a partir de la semilla 
del almendro se utilizó como materia prima semillas secas del fruto del almendro, sometidas a los siguientes tratamientos.

Determinaciónácidos grasos presentes. La muestra seca se sometió a extracción del aceite empleando como solvente hexano 96\%. La extracción del aceite se realizó mediante un montaje Soxhlet. Las hojuelas de almendra desengrasada, se sometieron a un secado leve para evaporar los residuos de hexano presentes. El secado se realizó en un horno (E\&Q) Electrónica y química).

\section{Análisis bromatológico. Métodos}

Determinación de humedad, 930.15/90 de la A.O.A.C adaptado (Bernal, 1993)

Determinación de grasa cruda o extracto etéreo, 920.39/90 A.O.A.C adaptado (Bernal, 1993)

Determinación de cenizas $942.04 / 90$ de la A.O.A.C adaptado (Bernal, 1993)

Determinación de fibra cruda 962.09/90 de la A.O.A.C adaptado (Bernal, 1993)

Determinación de Proteína cruda 955.04/90 de la A.O.A.C adaptado (Bernal, 1993)

\section{Obtención Harina de almendras con grasa y desgrasada}

Las semillas del fruto del almendro enteras y sin daños mecánicos, fueron laminadas, empleando una Laminadora de Cereales FDA-LAMCER-100. Se obtuvieron hojuelas delgadas de $1 \mathrm{~mm}$ aproximadamente. Estas fueron sometidas a un proceso de secado en un horno (E \& Q Electrónica y química) por un tiempo de 60 min a una temperatura de $100{ }^{\circ} \mathrm{C}$ hasta lograr disminuir la humedad presente en la muestra a 5\% aproximadamente. La muestra seca se sometió a extracción del aceite empleando como solvente hexano $96 \%$, en un dispositivo Soxhlet. Las hojuelas de almendra desengrasada, se sometieron a un secado para evaporar los residuos de hexano presentes, en un horno (E\&Q; Electrónica y química. Mod. MMW 2011). El producto seco desengrasado y no desengrasado se sometió a un proceso de molienda independientemente utilizando un Blender, procesador de cereales, (Simply, Modelo SLO9M. 2011). Se realizó un tamizado de la harina, tamices Tyler resultante del proceso de molienda, con la finalidad de obtener una cantidad homogénea de finos. Las harinas de almendras con grasa y sin grasa fueron empacadas, por separado avacío en bolsas de polietileno.

\section{Elaboración de Tortas a partir de Harina de Almendras}

Para la elaboración de las tortas se utilizó la formulación de una torta básica de harina de trigo, descrita en la Tabla 1. El proceso de elaboración de las tres tortas se desarrolló realizando el pesado de las materias primas de acuerdo a la formulación. Luego se realizó el mezclado y batido de la margarina de repostería y el azúcar. Después se llevó a cabo el mezclado del resto de ingredientes (harinas, polvos para hornear, sal y nuez moscada). Finalmente la mezcla de los ingredientes se vertió en los moldes de tal forma que se colocaron en las esquinas y en el centro de este, para luego esparcir suavemente en todo el molde. Las masas fueron llevadas en los moldes al horno a unos $150^{\circ}$ durante 40 minutos.

Tabla 1. Formulaciones utilizadas en la elaboración de las tortas.

\begin{tabular}{|l|c|c|c|}
\hline \multirow{2}{*}{ Ingredientes } & \multicolumn{3}{|c|}{$(\%)$ Formulación } \\
\cline { 2 - 4 } & $\begin{array}{c}\text { Torta de } \\
\text { trigo base }\end{array}$ & $\begin{array}{c}\text { Tortas de harina } \\
\text { de almendras }\end{array}$ \\
\cline { 2 - 4 } & $\begin{array}{c}\text { Con } \\
\text { grasa }\end{array}$ & $\begin{array}{c}\text { Sin } \\
\text { Grasa }\end{array}$ \\
\hline Azúcar & 80 & 80 & 80 \\
\hline Harina de trigo & 100 & 60 & 60 \\
\hline $\begin{array}{l}\text { Harina almendra } \\
\text { con grasa }\end{array}$ & 0,00 & 40 & 0,00 \\
\hline $\begin{array}{l}\text { Harina almendra } \\
\text { sin grasa }\end{array}$ & 0,00 & 0,00 & 40 \\
\hline Huevo & 100 & 100 & 100 \\
\hline Margarina & 80 & 80 & 80 \\
\hline Nuez moscada & 0,20 & 0,20 & 0,20 \\
\hline Polvo de hornear & 2,00 & 2,00 & 2,0 \\
\hline Sal & 0,30 & 0,30 & 0,30 \\
\hline
\end{tabular}

Fuente: Sena 2012

\section{Elaboración de almendras confitadas}

La obtención de almendras confitadas de la semilla del almendro se realizó utilizando bombo de grajeado del fabricante (Fedeacero Modelo WQA 2011). Se realizó un pre-recubrimiento de los núcleos o almendras, con jarabe de sacarosa pulverizada y gelatina. La relación- 
del pre recubrimiento con respecto a los núcleos fue de $5: 1 \mathrm{p} / \mathrm{p}$. El procedimiento consistió en adicionar los núcleos (Almendras) al bombo de grajeado, ajustando la inclinación del bombo a $45^{\circ}$ y las revoluciones a 30 rpm (Andréo et al., 2007). Luego se procedió a adicionar lentamente la solución (jarabe) de pre recubrimiento previamente preparado con el consiguiente secado de las almendras con aire caliente $\left( \pm 45^{\circ} \mathrm{C}\right)$. Se siguió adicionando solución de recubrimiento paulatinamente con posterior secado por suministro de aire. Finalmente se adicionó el polvo de recubrimiento (azúcar y almidón) y se secaron los núcleos a la temperatura anterior. Luego de la solución de pre recubrimiento, se realizó el engrosado y alisado de los núcleos, el primero se realizó con una solución de sacarosa con colorantes en una relación de 0.9:1 p/p, veces el peso de los núcleos y el alisado de los núcleos se realizó empleando jarabe USP con azúcar pulverizada disuelta en una relación de 1,9:1p/p, veces el peso de los núcleos. Ambas etapas fueron realizadas bajo el mismo procedimiento descrito anteriormente (pre-recubrimiento), excepto por la aplicación de polvos de recubrimiento y una inclinación del bombo de grajeado mayor.

\section{Análisis estadístico}

Se evaluó la aceptación o rechazo del producto empleando como instrumento de medición una escala hedónica verbal de 5 puntos. Asimismo el análisis estadístico de los datos obtenidos en las pruebas de aceptación en la elaboración de los 2 productos, se realizó mediante ANOVA y prueba de Friedman con comparación de medias (Tukey). Se realizó un análisis sensorial inmediatamente después de la elaboración de cada uno de los productos antes mencionados. Se empleó la prueba de medición del grado de satisfacción, en esta prueba afectiva se contó con 50 panelistas no en- trenados compradores del tipo de alimento en cuestión, cuyas edades estaban comprendidas entre los 16 y 50 años de edad, pertenecientes a la Universidad de Córdoba (Sede Berástegui y Montería) y posibles consumidores establecidos en la ciudad de Montería.

\section{Resultados y discusión}

\section{Obtención de Tortas a partir de Harina de Almendras}

Se obtuvo harina con grasa y sin grasa a partir de semillas de almendras, el contenido de humedad, proteína, carbohidratos y grasa en el producto final se muestran en la Tabla 2. El diámetro de partícula para la harina de almendras presentó tamaños comprendidos entre 500 y $250 \mu \mathrm{m}$, pasando el $70 \%$ por el tamiz de $250 \mu \mathrm{m}$. Los contenidos de grasa y humedad son factores importantes en el tratamiento de las semillas potencialmente destinadas al procesamiento industrial, debido a que son parámetros importantes en la conservación de la materia prima y además juegan un papel importante en el aprovechamiento tecnológico de la almendra. Asimismo el alto porcentaje en grasa cruda, ofrece la oportunidad de extraer el aceite, con la finalidad de aprovecharlo en la elaboración de productos farmacéuticos y cosméticos, (Arrazola et al., 2014).

Los beneficios del consumo de un producto con las características de las tortas obtenidas se encuentran asociados en parte al perfil nutricional de la almendra, en particular a sus contenidos en tocoferol, ácidos oleico, fitoesteroles y compuestos fenólicos, proteinas (Chen y Blumberg, 2008). Los valores de grasa encontrados en la semilla del almendrón, son muy parecidos a lo reportado en la tabla de composición de alimentos, para los frutos secos almendra y avellana $(54,1 ; 58,0$

Tabla 2. Contenido de Humedad y grasa de harinas de almendras

\begin{tabular}{|c|c|c|c|c|c|}
\hline \multicolumn{3}{|l|}{ Proteínas (g) } & $20,6 \pm 0,5$ & $\begin{array}{c}\text { Harina con } \\
\text { grasa }\end{array}$ & $\begin{array}{c}\text { Harina sin } \\
\text { grasa }\end{array}$ \\
\hline \multicolumn{3}{|c|}{ Carbohidratos (g) } & $5,43 \pm 0,5$ & & \\
\hline \multicolumn{3}{|l|}{ Fibra (g) } & $9,57 \pm 1,2$ & & \\
\hline \multicolumn{3}{|c|}{ Contenido graso total (g) } & & $51,2 \pm 0,8$ & $7,6 \pm 0,4$ \\
\hline \multirow{3}{*}{ Ácidos grasos } & \multicolumn{2}{|l|}{ Saturados (g) } & $4.46 \pm 0,2$ & & \\
\hline & \multirow{2}{*}{ Insaturados (g) } & Mono insaturados (g) & $42,38 \pm 0,1$ & & \\
\hline & & polinsaturados (g) & $11,16 \pm 0,1$ & & \\
\hline Humedad (\%) & & & & $9,20 \pm 0,7$ & $6,2 \pm 0,3$ \\
\hline
\end{tabular}

Fuente: Propia 
g/100g respectivamente) (Senser, y Heimo, 1991), cuyos beneficios han sido ampliamente estudiados, así como lo reportado en el almendrón, con valores que oscilan entre 52,95 y $57,96 \mathrm{~g} / 100$ gramos y con otros frutos secos como maní y nueces cuyos valores son 49,20; 61,90 g /100 g respectivamente (Nagappa et al., 2003). Dado el alto contenido de grasa lha, et al., (2014) presenta este fruto para la industrialización y obtención además de biocombustibles.

Proteína. Los resultados de esta investigación mostraron que los niveles de proteína cruda de esta semilla están por encima del contenido proteico reportado para cereales como el arroz, avena, cebada y maíz $(8,1 ; 14,7 ; 11,3$ y $9,0 \mathrm{~g} / 100 \mathrm{~g}$ respectivamente), son semejantes a alimentos de origen animal la carne de pollo y de cerdo $(14,0$ y $16,5 \mathrm{~g} / 100 \mathrm{~g}$ respectivamente) y con los frutos secos avellanas y nueces $(12,5 \mathrm{y}$ $14,0 \mathrm{~g} / 100 \mathrm{~g}$ respectivamente) (Nagappa et al., 2003). El contenido de proteínas de la almendra (Terminalia catappa) es similar al de las almendras del género Prunus amygdalus Miller, cuyo contenido es del 21,26\% Oliveira et al., (2000) y 11,52 Agunbiade y Olanlokun (2006)

Fibra. El contenido de fibra de la almendra se encontró entre el 9 y el $15 \%$, que al ser comparado con las almendras Prunus amygdalus (11,8\%), las avellanas $(9,7 \%)$ y las nueces $(6,7 \%)$ presenta un mayor contenido (Oliveira et al., 2000). Para evaluar la calidad de la harina de almendra se hicieron tortas mediante la sustitución parcial de harina de trigo por harina de almendras. Los productos elaborados se sometieron a un análisis sensorial por 50 catadores no entrenados, con la finalidad de evaluar el color, la apariencia y sabor de las tortas elaboradas con harina de almendras y de harina de trigo (Figura 1).
Los resultados obtenidos durante la evaluación sensorial de tortas de almendras, en cuanto a la apariencia en general el $54 \%$ de la población encuestada equivalente a 27 personas les gustó mucho y ligeramente la torta de almendras con grasa, mientras que el $40 \%$ equivalente a 20 personas les gustó mucho y ligeramente el tratamiento de torta hecha con harina de almendras desengrasada. Sin embargo fue el tratamiento control, es decir la torta hecha en un $100 \%$ con harina de trigo la de mayor preferencia en cuanto a apariencia para los catadores, con un $84 \%$ de me gusta mucho, Figura 2.

Por otro lado el resultado para el sabor de las tortas de almendras muestra que la torta de almendras con grasa presentó un 58\% de respuestas, en el rango de "me gusta mucho" y "me gusta ligeramente". Asimismo la torta de almendras sin grasa tuvo una aceptación por los catadores del $50 \%$ correspondientes a 25 personas que les gustó mucho y ligeramente el sabor. El tratamiento control sigue mostrando una alta aceptabilidad con un $84 \%$ de me gusta mucho.

Se encontraron diferencias estadísticamente significativas entre los tratamientos $(p<0,05)$ con respecto a la apariencia y el sabor de las diferentes tortas elaboradas (Figura 2). Para los bloques (Individuos) no existe una diferencia significancia entre lo que percibió cada uno con respecto a la apariencia. Lo cual indica que los tratamientos aplicados son muy similares y que no inciden en gran magnitud en el producto final, para que el consumidor pueda establecer una diferencia. Según los resultados arrojados en la prueba de TUKEY para comparación de medias de tratamiento, se encontró que tanto para la apariencia como para el sabor no hay diferencia significativa para los tratamientos hechos con harina desengrasada y con grasa de almendras cuyo porcentaje de harina fue del $37 \%$. Sin

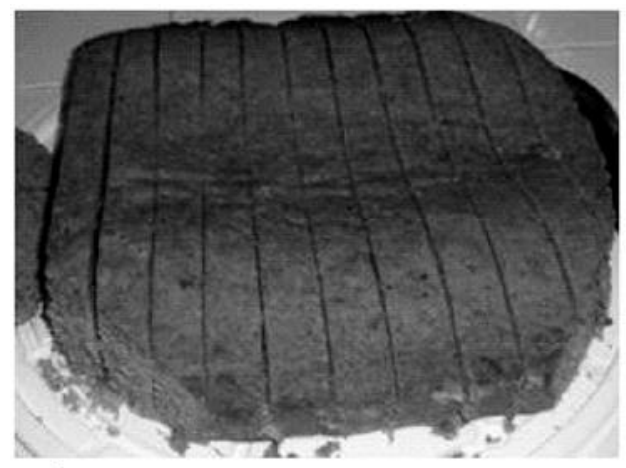

a)

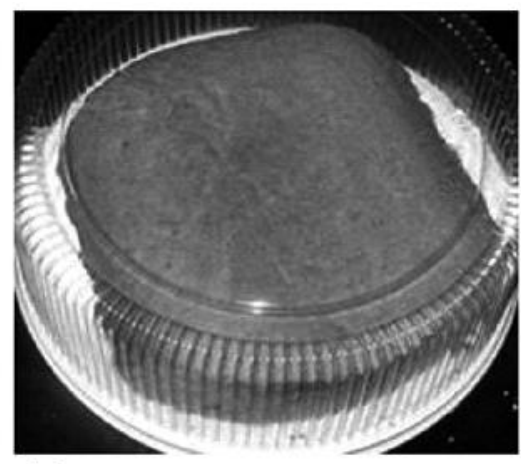

b)

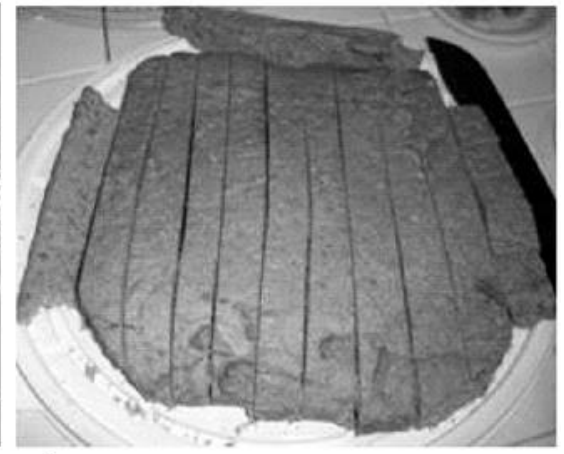

c)

Figura 1. Tortas de harina de almendras a y b) con grasa, c) sin grasa 


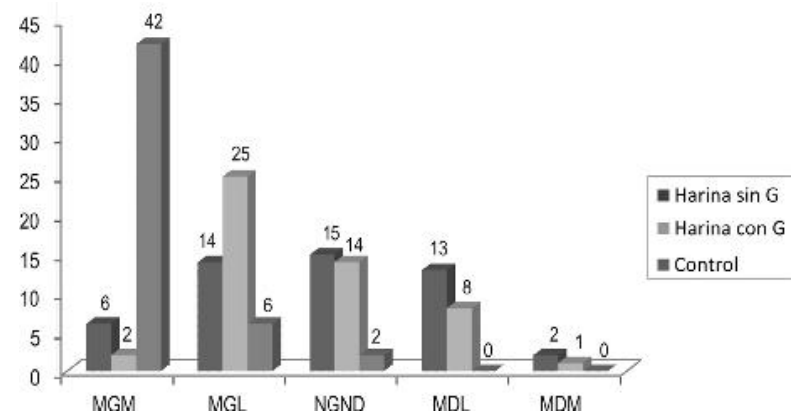

Figura 2. Resultados de apariencia general de tortas de harina de almendras

MGM Me gusta mucho

MGL Me gusta ligeramente

NGND Ni me gusta, ni me disgusta

MDL Me disgusta ligeramente

MDM Me disgusta mucho

embargo estos dos tratamientos difieren del control, hecho solo a base de harina de trigo. Es decir, existe un mayor grado de satisfacción por la torta de almendras hecha a base de harina de trigo por parte de los consumidores.

Los catadores hicieron comentarios referentes al color más oscuro de las muestras con harina de almendras, con respecto al control, este cambio en el color podría ser atribuido a la composición propia de la almendra, debido a las reacciones de Maillard entre los azúcares reductores y proteínas (Siddiq et al., 2009). Similares resultados observaron Surco y Alvarado (2011) al sustituir harina de sorgo por harina de trigo en productos de panadería, ya que a niveles superiores al 20\% hubo rechazo del producto por parte de los panelistas, mientras que el 5\% de ésta harina presentó buena aceptación sensorial y mejor color (Figura 3). El sabor de las tortas fue otro factor determinante, debido a que las tortas de almendras tenían un sabor diferente al control; en almendras de la India se han identificado varios compuestos responsables de su aroma y sabor, dentro de estos los furanos y compuestos heterocíclicos vo-

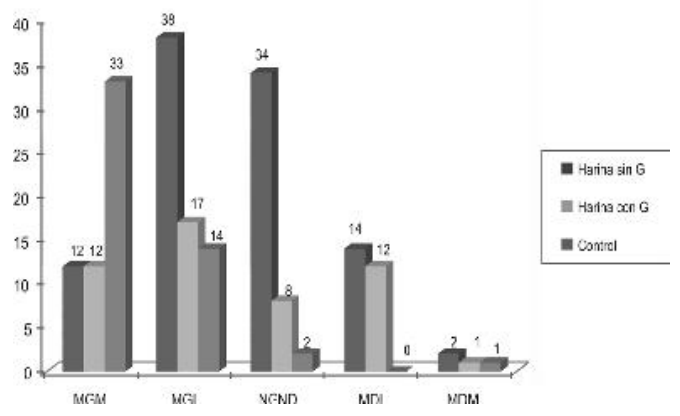

Figura 3. Resultados de sabor de tortas de harina de almendras látiles son los más representativos (Lasekan y Abbas, 2010). (Jia et al., (2008), realizaron tortas con harina de almendras Californiana, observaron que la sustitución de harina de almendra afectó todos los atributos sensoriales, de esta manera la intensidad del sabor de la almendra y el color aumentaron en el producto al incrementar la cantidad de harina de almendras.

Las tortas de almendras elaboradas con harina desengrasada presentaron una textura más dura que las muestras con harina de almendras sin desengrasar, de esta manera, la grasa aportada por la harina de almendra podría haber contribuido a la mayor suavidad de las tortas. Siddiq et al, (2009) observó que la dureza de la masa en la elaboración de panes se incrementó significativamente con el aumento del nivel de harina de almendras desengrasada en la mezcla, atribuyendo este hecho a la mayor capacidad de absorción de agua de la harina desengrasada.

El volumen de las tortas con harina de almendras desengrasada y con grasa fue menor con respecto a la torta de harina de trigo; Este hecho se encuentra claramente relacionado al efecto que produce una disminución de la cantidad de gluten en la mezcla, debido a que la cantidad de gluten que contiene la harina de trigo imparte un mayor volumen en torta (Pascual y Zapata, 2010; Siddiq et al., 2009). El porcentaje de harina de almendras (40\%), modificó los atributos sensoriales de la torta tradicional de harina de trigo. Jia et al., (2011), observó una disminución en las puntuaciones de todos los atributos sensoriales y la aceptabilidad global de galletas elaboradas con sustitución parcial de harina de trigo por harina de almendras, la máxima cantidad de harina de almendras en galletas aceptada sensorialmente fue de $20 \%$. Asimismo la adición de $20 \%$ de harina de almendras desengrasada a panes demostró tener un efecto negativo en los atributos sensoriales, siendo el 15\% de harina de almendras el que mejoró la aceptación sensorial (Siddiq et al., 2009). Sin embargo, Jia et al., (2008) reportó que tortas con 70\% de harina de almendra remplazada en la formulación tradicional era la más favorecida por el panel sensorial.

\section{Elaboración de Almendras Confitadas}

Se obtuvieron almendras confitadas recubiertas de varios colores las cuales fueron sometidas a análisis sensorial por 50 catadores no entrenados, quienes evaluaron la apariencia, el sabor y la textura de las almendras confitadas elaborada y de dos marcas comerciales de almendras (Marca 1 y Marca 2). La Figura, 4 muestra los resultados obtenidos durante la evaluación sensorial para tres tipos de almendras confitadas, observándose que en cuanto a la apariencia 
en general el $40 \%$ de los catadores equivalente a 20 personas les gustó mucho y ligeramente la almendra confitada mientras que el $88 \%$ equivalente a 44 personas y el 100\% (50 catadores) les gustó mucho y ligeramente las almendras confitadas de la marca comercial 1 y 2 respectivamente. Por otro lado el resultado para el sabor muestra que la almendras confitadas presentaron un $60 \%$ de me gusta mucho y me gusta ligeramente, que corresponde a un total de 30 personas. Las muestras comerciales 1 y 2 presentaron el 82 y $90 \%$ de me gusta mucho y ligeramente respectivamente, teniendo estos dos productos comerciales una alta aceptabilidad (Figura 5).

Asimismo la textura (Dureza) de las almendras se evaluó por análisis sensorial (Figura 6), los resultados muestran que la almendra confitada presentó un $50 \%$ de me gusta mucho y me gusta ligeramente, correspondiente a 25 personas. La almendra comercial de marca 1 presentó una aceptación de 62 \% y para la marca 2 una aceptación del $82 \%$ en me gusta mucho y ligeramente. El análisis estadístico muestra que hay diferencias estadísticamente significativas entre los tratamientos $(p<0,05)$ con respecto a la apariencia, el sabor y textura de almendras confitadas. Para los bloques (Individuos) no existe una diferencia significancia entre lo que percibió cada uno.

Según los resultados arrojados en la prueba de TUKEY para comparación de medias de tratamiento, se encontró que la apariencia y el sabor no hay diferencia significativa para las almendras confitadas comerciales

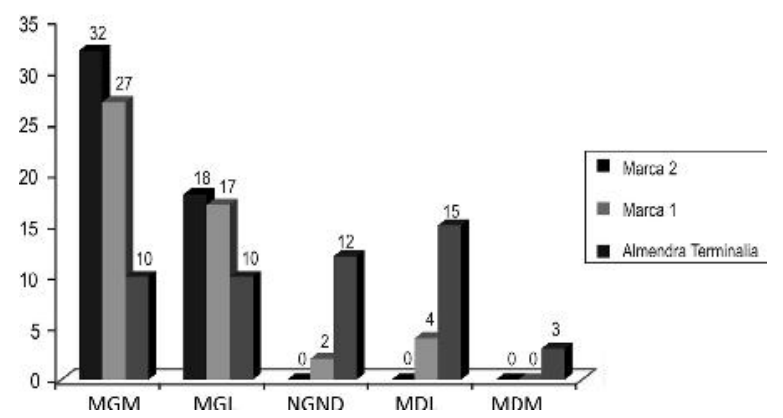

Figura 4. Resultados de apariencia Almendras confitadas

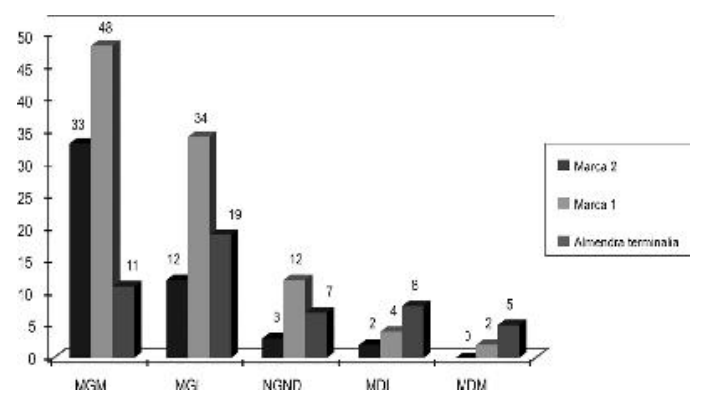

Figura 5. Resultados de Sabor Almendras confitadas

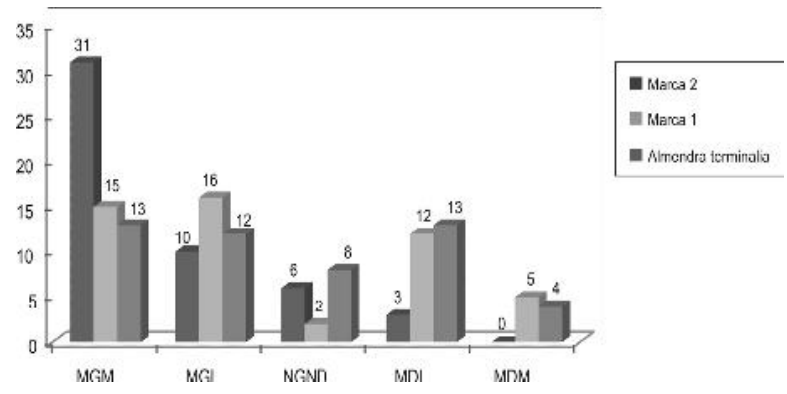

Figura 6. Resultados de dureza Almendras confitadas

Sin embargo estos dos tratamientos difieren de la almendra confitada Terminalia catappa. Existiendo un mayor grado de satisfacción por las almendras confitadas comerciales. Por otro lado los resultados arrojados en la prueba de TUKEY para comparación de medias de tratamiento con respecto a la textura (Dureza) de las almendras confitadas, arrojó que no hay diferencias significativas en la aceptabilidad demostrada por los catadores para la almendra confitada Marca 1 y la almendra confitada, siendo esta dos diferentes significativamente de la almendra de marca comercial 2.

Los catadores manifestaron que fue la falta de brillo, el parámetro que más afecto la apariencia general de la almendra confitada Terminalia catappa comparada con la marca 1 y 2. La falta de brillo, junto a la superficie no uniforme (piel de naranja) son defectos tecnológicos importantes en el confitado de almendras; los cuales son consecuencias del sistema de secado y de la temperaturas del mismo de manera que debe existir un equilibrio entre la cristalización de las capas y el secado; de manera que cada una de las capas haya fraguado antes de la aplicación de la capa siguiente (Nurmi et al., 2005). Los atributos de sabor, textura y apariencia en general evaluados en las almendras confitadas mostraron una buena aceptación sensorial aunque con diferencias significativas con respecto a las marcas comerciales, sin embargo la optimización de las formulaciones y la estandarización del proceso podrían mejorar los atributos sensoriales del producto. Otro punto destacable es su contenido en fibra. A pesar de su aspecto seco y homogéneo, la almendra posee una cantidad notable de fibra soluble (10\%). Agunbiade y Olanlokun (2006), reportaron valores de fibra del orden 5,09\%, Proteína de $11,52 \%$ muy por debajo de los resultados obtenidos en esta investigación mencionados anteriormente.

\section{Conclusiones}

La apariencia y el sabor de tortas de harina de almendras desengrasada y con grasa no presentaron diferencias significativas en cuanto a las percepciones de los catadores; no obstante, estos dos tratamientos fueron diferentes al control, siendo estos productos de mejor 
aceptación sensorial. Las almendras confitadas Terminalia catappa presentaron una textura crujiente, detectada por los catadores similar a almendra confitada de la marca comerciales hoy. Las almendras confitadas y tortas de harina de almendras presentaron buena aceptabilidad por los consumidores de estos productos, siendo una alternativa de aprovechamiento industrial y nutricional. El estudio demostró que la almendra (Terminalia catappa) ofrece un gran potencial para ser utilizado en una variedad de productos alimenticios, siendo viable para el desarrollo de nuevos productos.

\section{Referencias}

Agunbiade S, Olanlokun J. Evaluation of some nutritional characteristic of Indian (Prunus amygdalus) Nut. Pakistan journal of nutrition. 2006;5(4): 316-318.

Ando M, Ito R, Ozeki Y, Nakayama Y, Nabeshima T. Evaluation of a novel sugar coating method for moisture protective tablets. Int J Pharm. 2007; 336:319-328.

Andréo N, Pessole L, Yang M, Issa M, Ferraz H. Aplicación de Recubrimiento Gastro-resistente en Núcleos Comprimidos conteniendo Didanosina utilizando Diferentes Equipos: Bombo Grageador Convencional, Tambor Perforado y Lecho Fluido. Lat Am J Pharm. 2007; 26(5): 669-676.

Association of Official Analytical Chemists. Official methods of analysis of the association of official analytical chemists. 11a ed. Washington: AOAC; 1970.

Arrázola G, Buelvas H, Arrieta Y. Nutritional characteristics of the indian almond (Terminiala catappa L.) as supplement in animal feeding. Revista MVZ Cordoba. 2008; 13(1): 1205-1214.

Arrázola G, Páez M, Alvis A. Composición, Análisis Termofísico y Análisis Sensorial de Frutos Colombianos. Parte 1: Almendro (Terminalia Catappa L.). Información Tecnológica. 2014;25(3):17-22

Chen C, Blumberg J. Phytochemical composition of nuts. Asia Pac J Clin Nutr. 2008;17(suppl 1):329-332.

Fasoli E, D'Amato A, Kravchuk A, Citterio A, Righetti G. In-depth proteomic analysis of non-alcoholic beverages with peptide ligand libraries. I: Almond milk and orgeat syrup. J. Proteomics. 2011;74:1080-1090.

González-MM, Mendoza F, Mora J, Mendoza M, Márquez J, Bravo B. Valor nutricional de la semilla del almendrón (Terminalia catappa Linn). Revista de la facultad de farmacia. 2005; 47(1):25-29.

Henao S, Aristizábal J. Influencia de la variedad de yuca y nivel de sustitución de harinas compuestas sobre el comportamiento reológico en panificación. Revista Ing Inv. 2009; 29(1): 39-46.

Hernández M, García L, Rojo D, Olivares D. Almendro de la India. Potencial biológico valioso. Rev Cubana Invest Biomed. 2003; 22(1):41-47.

Jia C, Huang W, Abdel-Samie MA, Huang G, Huang G. Dough rheological, Mixolab mixing, and nutritional characteristics of almond cookies with and without xylanase. J. Food Eng. 2011;105: 227-232.

Iha O, Alves F, Suarez P, Silva C, Meneghetti M, Meneghetti S. Potential application of Terminalia catappa L. and Carapa guianensis oils for biofuel production: Physical-chemical properties of neat vegetable oils, their methyl-esters and bio-oils (hydrocarbons). Ind Crop Pro. 2014;52: 95-98

Jia C, Kim YS, Huang W, Huang G. Sensory and instrumental assessment of Chinese moon cake: Influences of almond flour, maltitol syrup, fat, and gums. Food Res Int. 2008;41:930-936.

Lasekan O, Abbas K. Analysis of volatile flavour compounds and acrylamide in roasted Malaysian tropical almond (Terminalia catappa) nuts using supercritical fluid extraction. Food Chem Toxicol. 2010; 48:2212-2216.

Matos L, Nzikou J, Kimbonguila A, Ndangui C, Pambou-Tobi N, Abena A, Silou Th, Scher J, Desobry S. Composition and Nutritional Properties of Seeds and Oil From Terminalia catappa L. Adv J Food Sci Technology. 2009; 1(1): 72-77.

Mejorado N. Confitería. Alfa Editores Técnicos. 2006; 28(2):10- 17.

Mejorando MN. 2006. Confitería. Industria alimentaria

Nagappa A, Thakurdesai P, Jiwan S. Antidiabetic activity of Terminalia catappa Linn fruits. J Ethnopharmacol. 2003; 88: 45-50.

Nurmi J, Pepper T, Oravainen J, Bond M, Fairs I. 2005. Procedimiento para el recubrimiento duro de núcleos de artículos masticables y núcleos producidos mediante el proceso. Patente Europea, 2232 592: 1-13.

Oliveira J, Vasconcelos I, Bezerra C, Silveira S, Monteiro A, Moreira A. 2000. Composition and nutritional properties of seeds from Pachira aquatica Aubl, Sterculia striata St Hil et Naud and Terminalia catappa Linn. HYPERLINK "http://www.sciencedirect. com/science/journal/03088146" \o "Go to Food Chemistry on ScienceDirect" Food Chem. 2000;70(2): 185-191. HYPERLINK "http://dx.doi.org/10.1016/S0308-8146(00)00076-5" \t "doilink" doi:10.1016/S0308-8146(00)00076-5

Onwulata C. 2005. Encapsulated and Powdered Foods. Primera Edición. Editorial CRC Press.

Pascual C, Zapata J. Sustitución parcial de harina de trigo Triticuma estivum L. por harina de kiwicha Amaranthus caudatus L. usando el método directo esponja y masa, en la elaboración de pan. Rev Soc Quím. Perú. 2010; 76(4):377-388.

Senser F, Heimo S. 1991. Tablas de composición de alimentos. El pequeño Sauci-Fachman-Kraut. Acribia Zaragoza. 96-97.

Siddiq M, Nasir M, Ravi R, Butt M, Dolan K, Harte J. Effect of defatted maize germ flour addition on the physical and sensory quality of wheat bread. LWT - Food Sci Technology. 2009;42:464-470.

Surco AJ, Alvarado KJ. Estudio Estadístico de Pruebas Sensoriales de Harinas Compuestas para Panificación. Rev Bol Quim. 2011; 28(2):79-82.

Roquette F, Cabinet P. 2006. Procedimiento mejorado para el recubrimiento duro de grageas. Traducción de Patente Europea, 2 249 611: 1-5. 